en valeur de nos rivières alpines dont quelques-unes étaient fort appauvries, a donné les meilleurs résultats et permet aujourd'hui d'en retirer le maximum de rendement avec le minimum de sacrifices.

\title{
ENCORE UN POISSON D'ÉLITE MENACÉ DANS LE LAC LÉMAN
}

\author{
Par M. R. VILLAUME \\ Inspecteur des Eaux et Forèts.
}

Après mon long cri d'alerte de l'an dernier en faveur de l'Omble-Chevalier, je me permettrai une nouvelle intervention en faveur de la Truite dite de lac. Le cri sera moins angoissé car, grâces leur soient rendues, nos excellents voisins et amis les Suisses, en particulier les pisciculteurs officiels du canton de Vaud, agissent avec un tel bonheur que leur action limite, pour le moment du moins, les effets du mal causé au peuplement du lac Léman, richesse mitoyenne, par quelques équipages de pêcheurs français. - Je dois ajouter ensuite, par gout de vérité, que ces pêcheurs ne doivent pas assumer la responsabilité toute entière de cette situation, il y a lieu de tenir également, pour partiellement responsables, non seulement les maladies de la Truite, mais encore tous les usiniers propriétaires de barrages infranchissables disséminés, en France comme en Suisse, sur les rivières tributaires du Lac Léman, mais j'estime que la part de responsabilité de ces usiniers $n$ 'est malgré tout qu'indirecte en quelque sorte et, en tout cas, non essentielle, car ils agissent surtout dans l'intérêt primordial des populations tandis que les pêcheurs agissent, avec un indéniable courage il est vrai, mais dans leur seul intérêt rigoureusement personnel et momentané contre 'intérêt professionnel et permanent de toute la population des pêcheurs, eux compris.

Je commencerai d'abord par quelques tableaux statistiques donnant l'importance relative des pêches de Truite dans le Léman. Divers lacs alpins seront étudiés plus loin.

\section{Lac Léman.}

Surface totale : 58.300 hectares, près de go millions mètres cubes d'eau. Eaux françaises : $42 \%$ - eaux vaudoises : $50 \%$ - eaux genevoises : $6 \%$ - eaux valaisannes : $2 \%$.

(1) Voir Bulletin : $-n^{\circ} 97$, Juillet 1936, p. $5 ;-n^{\circ}$ 98, Aoùt, p. 33 . 
La peche de la Truite a toujours été très varî̉able en quantité dans des propartions très grandes. - Aussi; jusqu'en rg25, nous bornerons-nous à donner, en ce qui concerne d'ailleurs les eaux françaises uniquement et ce sur le vu des renseignements bénévoles fournis par le commerce en poissons, les points bas et les points hauts de la courbe :

\begin{tabular}{|c|c|c|}
\hline ANAEES & POIDS DE TRUITE & \\
\hline $\begin{array}{l}1897 \\
1900 \\
1902 \\
1904 \\
1905 \\
1908 \\
1912 \\
1914 \\
1919 \\
1920 \\
1923 \\
1925\end{array}$ & $\begin{array}{r}4.769 \mathrm{kgs} \\
8.139- \\
4.750- \\
11.553- \\
6.179- \\
20835- \\
8.191- \\
13.714-(1) \\
12.346- \\
5439- \\
3.350- \\
19.558-(2)\end{array}$ & $\begin{array}{l}\text { (1) Deux trimestres seulement. } \\
\text { (2) Burant cette saison 1925, it y a } \\
\text { ou une épidémie grave sur la Perche } \\
\text { dont les captures ont baiss de de } \\
106.000 \mathrm{kgs} \text { en 1924, a } 83.600 \mathrm{kgs} \text {. }\end{array}$ \\
\hline
\end{tabular}

De I926 à 1936, nous croyons utile de donner des renseignements un peu plus détaillés par trimestre, tant pour Jes-eaux françaises que pour les eaux vaudoises; depuis 1932 pour les eaux genevoises.

\begin{tabular}{|c|c|c|c|c|c|c|c|}
\hline \multicolumn{8}{|c|}{ WAUX FRATUAIEMS } \\
\hline \multirow{2}{*}{ AMNEES } & \multicolumn{4}{|c|}{ POIDS EN KILOS DE TRUI IES FECAÉES } & \multirow{2}{*}{$\begin{array}{c}\text { TOTAL } \\
\text { TRurris - } \\
\end{array}$} & \multirow{2}{*}{$\begin{array}{l}\text { Total } \\
\text { en kgs } \\
\text { de poinsons } \\
\text { plehés }\end{array}$} & \multirow{2}{*}{$\%$} \\
\hline & 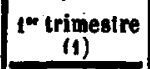 & trimestre & 3* trimestre & 40 trimestre & & & \\
\hline \multirow[t]{2}{*}{$\begin{array}{l}1926 \\
1927 \\
1928 \\
1929 \\
1930 \\
1931 \\
1932 \\
1933 \\
1934 \\
1935 \\
1936\end{array}$} & $\begin{array}{l}6.414 \\
6.295 \\
4.747 \\
5.111 \\
3.385 \\
4.470 \\
6.385 \\
4.072 \\
2.988 \\
5.289 \\
2.844\end{array}$ & $\begin{array}{l}2.490 \\
1.752 \\
1.331 \\
1.894 \\
1.591 \\
1.365 \\
1.728 \\
1.251 \\
.749 \\
739 \\
710\end{array}$ & $\begin{array}{l}3.998 \\
2.580 \\
3.365 \\
2.348 \\
3.253 \\
3.477 \\
3.178 \\
2.390 \\
3.000 \\
1.424 \\
1.324\end{array}$ & $\begin{array}{l}2.280 \\
4.278 \\
3.191 \\
3.448 \\
6.217 \\
3.151 \\
3.422 \\
2.816 \\
2.137 \\
4.071 \\
2.486\end{array}$ & $\begin{array}{r}15.182 \\
14.905 \\
12.634 \\
12.801 \\
14.446 \\
12.463 \\
14.713 \\
10529 \\
8.874 \\
11.617 \\
7.364\end{array}$ & $\begin{array}{r}201.100 \\
-180.700 \\
182.600 \\
329800 \\
395.500 \\
286.200 \\
304.000 \\
284.600 \\
-318.100 \\
290.000 \\
280.600\end{array}$ & $\begin{array}{l}7,5(6,4) \\
8,2(6,0) \\
6,9(5,2) \\
3,8(2,8) \\
3,8(2,1) \\
4,3(3,2) \\
4,8(3,7) \\
3,7(2,7) \\
2,8(2,1) \\
4,4(2,0) \\
2,6(1,7)\end{array}$ \\
\hline & 52.000 & 15.594 & 30.397 & 37.497 & 135.428 & 3.023700 & \\
\hline \multicolumn{5}{|c|}{ 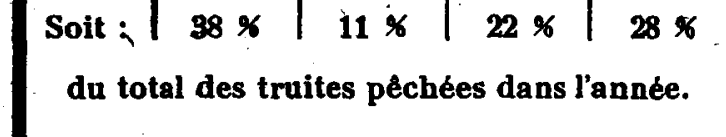 } & \multicolumn{3}{|c|}{$\begin{array}{l}\text { Soit en moyenne } 4,4 \% \text {, } \% \\
\text { Sans le } 4 \circ \text { trimestre : } 3,2 \% \text {, } \\
\text { movennes entre parentheses } \\
\text { derniere colonne.- }\end{array}$} \\
\hline
\end{tabular}

(r) En presqué totalité résultent des prises en pechè de lac. 


\begin{tabular}{|c|c|c|c|c|c|c|c|}
\hline \multirow{3}{*}{ ANNÉES } & \multicolumn{7}{|c|}{ HAUX VAUDOISES } \\
\hline & \multicolumn{4}{|c|}{ POIDS EN KIL,OS DE TREITES PÊCHÉES } & \multirow{2}{*}{$\begin{array}{c}\text { TOTAL } \\
\text { TnEITES }\end{array}$} & \multirow{2}{*}{$\begin{array}{c}\text { TUTAL } \\
\text { en kgs } \\
\text { de poissons } \\
\text { pèches }\end{array}$} & \multirow{2}{*}{$\%$} \\
\hline & $1 \bullet$ trimestre & $2 \cdot$ trimestre & $3 \cdot$ trimestre & to trimestre & & & \\
\hline $\begin{array}{l}1926 \\
1927 \\
1928 \\
1929 \\
1930 \\
1931 \\
1932 \\
1933 \\
1934 \\
1935 \\
1936\end{array}$ & $\begin{array}{r}168 \\
162 \\
139 \\
50 \\
61 \\
209 \\
108 \\
56 \\
105 \\
127 \\
118\end{array}$ & $\begin{array}{l}877 \\
807 \\
593 \\
393 \\
566 \\
375 \\
444 \\
432 \\
408 \\
467 \\
477\end{array}$ & $\begin{array}{ll}5 & 400 \\
1 & 938 \\
2.845 \\
1.4 \times 5 \\
1 & 846 \\
1.366 \\
1 & 846 \\
1 & 933 \\
1.628 \\
1.815 \\
1 \quad 636\end{array}$ & $\begin{array}{r}45 \\
" \\
" \prime \\
30 \\
\prime \prime \\
12 \\
\prime \prime \\
" 1 \\
11 \\
16 \\
14\end{array}$ & $\begin{array}{ll}6.489 \\
2 . & 80 \\
3 & 579 \\
2 & 066 \\
2 & .901 \\
2 & .567 \\
3 & 356 \\
3 & 543 \\
3 & 052 \\
3 & 286 \\
3.122\end{array}$ & $\begin{array}{r}106.000 \\
259.800 \\
116.700 \\
89.600 \\
142.100 \\
139.200 \\
126.600 \\
141.700 \\
146.200 \\
139.700 \\
150.200\end{array}$ & $\begin{array}{l}7,3 \\
2,7 \\
3 \\
2,5 \\
2 \\
2 \\
2,5 \\
2,5 \\
2,1 \\
2,3 \\
2,1\end{array}$ \\
\hline $\begin{array}{l}\text { Soit : } \\
\text { du to }\end{array}$ & $\begin{array}{c}1303 \\
3,5 \% \\
\text { des tru }\end{array}$ & $\begin{array}{c}5.841 \\
15,8 \% \\
\text { tes pêché }\end{array}$ & $\begin{array}{c}23789 \\
64,3 \stackrel{\circ}{~} \\
\text { es dans l' }\end{array}$ & $\begin{array}{c}128 \\
0,3 \%\end{array}$ & \multicolumn{3}{|c|}{ Soit : $2,3 \%$ en moyenne. } \\
\hline $\begin{array}{r}\text { No } \\
\text { provie }\end{array}$ & $\begin{array}{l}\text { La diff } \\
\text { prise's }\end{array}$ & $\begin{array}{l}\text { ce entre } \\
\text { traineur }\end{array}$ & $\begin{array}{l}\text { tal dor } \\
\text { ateurs }\end{array}$ & $\begin{array}{l}\text { la so } \\
\text { du tol }\end{array}$ & $\begin{array}{l}\text { des chi } \\
\text { prises }\end{array}$ & s trimestri & $\mathrm{kgs})$. \\
\hline
\end{tabular}

\begin{tabular}{|c|c|c|c|c|c|c|c|}
\hline \multicolumn{8}{|c|}{ EAUX } \\
\hline \multirow{2}{*}{ ANNÉES } & \multicolumn{4}{|c|}{ POIDS EN KHIOS DE THCITES PÉCHÉES } & \multirow{2}{*}{$\begin{array}{l}\text { TOlAL, } \\
\text { TrutTES }\end{array}$} & \multirow{2}{*}{$\begin{array}{c}\text { Toral } \\
\text { en kgs } \\
\text { de poissons } \\
\text { péchès }\end{array}$} & \multirow{2}{*}{$\%$} \\
\hline & $1 \cdot r$ trimestre & $z^{*}$ trimestre & $3 \cdot$ trimestre & 4. trimestre & & & \\
\hline \multirow[t]{2}{*}{$\begin{array}{l}1932 \\
1933 \\
1934 \\
1935 \\
1936\end{array}$} & $\begin{array}{r}11 \\
5 \\
15 \\
5 \\
15\end{array}$ & $\begin{array}{l}21 \\
18 \\
32 \\
34\end{array}$ & $\begin{array}{r}27 \\
21 \\
21 \\
2 \\
7\end{array}$ & $\begin{array}{l}\eta \\
2 \\
1 \\
1 \\
\eta\end{array}$ & $\begin{array}{l}71 \\
56 \\
35 \\
40 \\
56\end{array}$ & $\begin{array}{l}19500 \\
1880 \\
17500 \\
16.900 \\
16200\end{array}$ & \begin{tabular}{l}
\multicolumn{1}{c}{)} \\
0,2 \\
0,31 \\
0,23 \\
0,34
\end{tabular} \\
\hline & 40 & 108 & 55 & 4 & 207 & 69400 & \\
\hline
\end{tabular}

Enfin, voici quelques renseignements concernant la pêche dans les eaux valaisannes :

En 1932 - pèché : $2 / 46 \mathrm{kgs}$ de truites sur un total de I $2.808 \mathrm{kgs}$ de poissons.

En r9.33 - pèché : $180 \mathrm{kggs}$ de truites sur un total de $9.250 \mathrm{kgrs}$ de poissons.

En r 934 -s 935 - les chiffres manquent.

En 1936 - pêché dans le $\mathrm{i}^{\text {er }}$ trimestre $9^{3} \mathrm{kgs}$ de Truites.

Iu total : 8.r4j $\mathrm{kg}$ go de poissons pêchés dans l'année.

Ces tableaux statistiques appellent quelques observations : 
Tableau des pëches Eaux françaises. - Les chiffres de pourcentage entre parenthèse donnent les renseignements concernant les trois premiers trimestres seuls, à l'exclusion du quatrième (en fait, d'ailleurs, constitué par les dix jours de lève). Ce sont les pourcentages entre parenthèse qui sont à comparer avec les chiffres suisses.

Si en moyenne sur les onze dernières années la truite ne représente que $4,4 \%$ du poisson pêché dans les eaux françaises, $1,2 \%$ se trouve pêché en fait en ro jours de Décembre.

Les renseignements portés sont ceux figurant aux relevés officiels annuels (les chiffres sont fournis bénévolement par les commerçants en poissons).

Tableau des pêches Eaux vaudoises. - C'est à l'extrême complaisance du Service forestier vaudois, en particulier à M. l'Inspecteur cantonal Grivaz et à M. l'Ingénieur S. Combe, que je dois les renseignements à peu près inédits qui figurent. Je m'excuse d'avoir parfois simplifié leurs documents qui comportaient des précisions non conservées.

J'attire l'attention sur le fait que des arrondissements peuvent laisser croire à des erreurs dans certaines additions, les totaux sont ceux approchés au plus juste. - De plus les produits de la pêche des pêcheurs amateurs ne peut être répartie trimestriellement, les poids figurent dans les totaux et ne sont connus qu'une fois par an. Pour la période considérée, les amateurs ont tiré de l'eau $\mathbf{5} .888$ kilogs, soit $15,9 \%$ environ du total.

Tableau des péches Eaux genevoises. - Les renseignements sont dûs à l'obligeance de M. W. BorkL, Inspecteur des Forêts du canton de Genève que je tiens à remercier.

Tableau des pêches Eaux valaisannes. - Les quelques renseignements reçus proviennent d'une communication de $M$. l'Inspecteur cantonal LoRETAN après intervention de M. DELAcoste, Inspecteur forestier à Monthey. Je suis heureux de les remercier ici.

Dans les lacs, il est pêché en fait de Truite à peu près exclusivement de la "Truite de lác ", ce n'est pas là une affirmation naĩve, beaucoup d'auteurs considèrent en effet la "Truite de lác " comme une espèce suffisamment distincte de la Truite de ruisseau pour avoir son nom et sa place à part dans la classification des Salmonides. La plus importante référence est celle de LinNE, qui l'a nommée Trutta lacustris, et nombreux sont ceux, moins qualifiés que lui, qui la distinguent des autres en basant cette discrimination sur des caractères' le plus souvent extérieùrs, ils séparent imperturbablement " la Truite commune " qui ne serait que la Truite des eaux courantes, de la "Truite argentée ", de la "Truite saumonée ", etc., etc...

Mais ce n'est pas l'opinion générale actuelle : Fatio se borne à en faire une forme à part (forma major) par opposition à forma minor des Truites de ruisśeaux ; nous estimons qu'il a parfaitement raison.

Placées dans des obnditions de vie analogues, toutes les Truites fario, quelle que soit l'origine des œufs, donnent des poissons identiques (qualité 
individuelle de sélection à part). - Les réactions sanguines sont les mêmes; Neresheimer voit, dans la Truite de lac, celle de ruisseau et même celle de mer, une seule et même espèce à variétés.

Une observation concluante est toute récente :

Au cours d'une pêche exceptionnelle, en Novembre 1936 , M. le Conservateur Martin a sorti de l'eau dans le lac artificiel de Bissorte, créé il y a deux ou trois ans, un total de I I pièces pesant 5 kilos. Le fond du Lac était tourbeux pour partie pour le reste formé d'anciens alpages; sur les I I Truites 2 étaient des Truites communes à livrée noire, points rouges, de grosse taille, l'une d'elle à forte tête - Une autre Truite également grosse, mais très grasse portant encore des points rouges sur une livrée jaunâtre - 8 enfin, les plus petites mais bien en chair pourtant, avaient la livrée argentée, à points noirs seuls, des Truites de lac.

Il est certain qu'aucun déversement de truite, à plus forte raison de "Truite de lac " n'a été fait dans ce réservoir ; les truites sont donc des anciens habitants des ruisseaux de Bissorte ; elles ont bien grossi vu l'importance et la qualité de la nourriture mise tout à coup à leur disposition. Les plus âgées, déjà mures sexuellement, ont conservé leur livrée première, la troisième, sans doute une truite un peu plus jeune, est en voie d'évolution de couleur ; les autres, des jeunes truites se' sont trouvées dans les conditions des truites dans les lacs et ont pris tout naturellement la livrée des truites de lac.

Ceci dit, décrivons ce beau poisson :

Salmonide, il possède tous les caractères du groupe, mais sa livrée est bien spéciale : d'une teinte généralement claire donnant sur le bleu argenté plus ou moins prononcé, surtout sur le dos et le haut des flancs; elle ne porte généralement que des taches noires étailées.

On a pu, valablement, séparer les truites de lac en deux catégories : - Les unes dites "Truites de fond " sont de forte taille souvent plusieurs kilos, ont une livrée relativement peu brillante, ont une queue à bord rectiligne : elles sont toujours difficiles à pêcher, ce sont les "Grund Forellen " des auteurs allemands. Les autres, beaucoup plus nombreuses, sont des "Truites argentées " "Schwebe Forellen ", à livrée vraiment claire, ne pesant jamais plus de 5 kilos (souvent beaucoup moins) et qui ont toujours une queue à bord échancré ; elles habitent surtout les eaux superficielles. Ce sont ces dernières qui ont eu la réputation d'être stériles. Je reviendrai à ce sujet plus loin et assez longuement. Ce sont, en tout cas, les unes et les autres, d'excellents poissons à chair souvent saumonée, pouvant atteindre 15 kilos assez fréquemment (on signale des prises de 3o kilos) en Truites de fond.

Les règlements en vigueur en France et valables pour les eaux françaises stipulent que la pêche est ouverte pour la Truite toute l'année à l'exception 
de la période du r $^{\text {er }}$ Octobre au 20 Décembre. Dans les eaux suisses, l'interdiction porte sur tout le quatrième trimestre entier.

Ces dates n'ont pas toujours été celles légales.

La convention franco-suisse de 1880 prévoyait une fermeture du ro Octobre au 20 Janvier; c'est l'arrêté suisse du 13 Août 1891 qui a établi la période telle qu'elle est, elle a été conservée dans la convention francosuisse de rgo4 et encore une fois consacrée en Suisse en rgi i, lors de la dénonciation de la convention.

Côté français : d'abord de 1880 à 1911 , la réglementation fut à ce sujet la même qu'en Suisse. Après la dénonciation, à partir de r9ı2, ce furent tout de même les dispositions de la convention qui restèrent appliquées et ce avec toutes les modifications qui furent apportées depuis rg18, soit par décisions préfectorales, soit par arrêtés ministériels et qui autorisèrent dès lors. la pêche de la Truite à partir du 20 Décembre. Le décret de I929 a sanctionné cette façon de faire et la situation est celle exposée plus haut.

En ce qui concerne la réglementation de la dimension des mailles des filets, elle est très complexe en France où intervient la grandeur même de l'engin ; à vrai dire, la fixation de cette dimension n'est que secondaire puisqu'elle fait en quelque sorte double emploi avec la question de dimension minima du poisson pêchable et vendable ; en général, toutefois, la pêche de la Truite se fait à la maille de $40 \mathrm{~m} / \mathrm{m}$. La dimension minima légale pour la Truite du lac fut d'abord fixée en France à $20 \mathrm{~cm}$.; de l'œeil à la naissance de la queue, cette longueur fut portée à $25 \mathrm{~cm}$. (longueur totale) en rgoh, puis à $30 \mathrm{~cm}$. en Suisse à partir de I9 I I - dimension fixée également en France, mais à partir de 1925 seulement par arrêté minis-, tériel. C'est encore la mesure réglementaire dans les deux pays que ne sépare plus somme toute que la question de la pêche de lève durant l'hiver (fin Décémbre surtout).

La Truite se pêche en effet, en hiver, d'une manière qui lui est bien spéciale : des filets araignées à maille de $40 \mathrm{~m} / \mathrm{m}$. au moins et de $2 \mathrm{~m}$. 80 de hauteur au plus, sont tendus à fleur d'eau la nuit en plein lac, accouplés par I5 au maximum. Par les nuits sans lune, ils capturent des quantités de Truites de la catégorie argentée ; les chiffres indiqués plus haut montrent que $28 \%$ des Truites pêchées dans les eaux françaises annuellement le sont durant ces dix jours. - Les poissons pêchés atteignent rarement le kilo et la moyenne de poids se balance vers la livre.

En dehors de ce mode, la Truite se prend à la ligne de fond, à la ligne traînante (amateurs) et, naturellement, dans les grands filets a raignées servant aussi pour les Ombles, les Corégones et la Perche (grands et petits pics). Il s'en capture aussi un certain nombre dans le grand filet (40 m. de hauteur, 120 mètres de longueur de bras), engin traîné, spécialement adapté à la capture des Corégones. Ces Truites sont alors généralement de très belles pièces. 
Est-il possible d'évaluer ce que représente en nombre de pièces les poids indiqués plus haut? Avec certitude non, mais, en restant dans le domaine des approximations, il est possible de donner un ordre d'idées. Le premier trimestre, en France comme en Suisse, les captures sont surtout du type des Truites pêchées de lève dont la moyenne est de 5oo grammes.

En onze ans, en France, comme en Suisse : 53.300 kilos ont été pêchés,

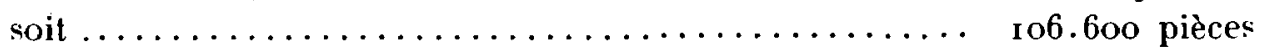

En été, la moyenne approche le kilog : $7 \overline{0} .000$ kilogs

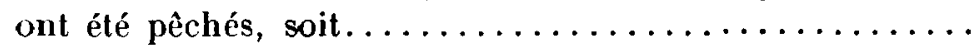

Pendant la dernière décade de Décembre : 37.500 kilogs

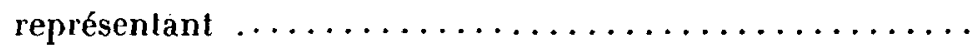

Enfin, les pècheurs amateurs suisses pêchent un poisson que l'on peut évaluer à 700 grammes de moyenne,

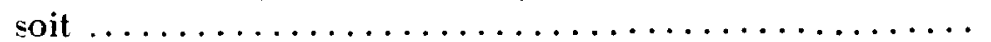

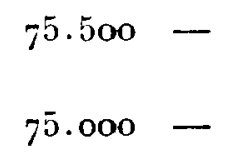

Au total en onze années.....

En chiffre très approché : 24.000 par an, pour plus de sûreté, nous dirons entre 20 et 30.000 pièces.

Pour rétablir la balance favorable, il faut donc au moins prévoir le remplacement de ces 20 à 30.000 poissons, il n'est pas exagéré de dire, vu les aléas que supportent les jeunes Alevins et les Truitelles dans le Lac, que c'est bien près de ro fois ce chiffre dont l'apport naturel ou artificiel doit être assuré, bornons-nous à envisager cette fourniture de 200.000 Alevins, ce chiffre nous permet de ne pas tenir compte de ce qui pourrait être pêché dans les eaux genevoises et valaisannes.

Le repeuplement en Truite du lac se fait par le jeu de la descente au lac des Alevins et Truitelles provenant des frayères sur lesquelles en hiver. dans les petits ruisseaux affluents du lac, sont allés frayer les reproducteurs sexuellement mûrs ; le frai est déposé dans les mêmes conditions que pour la Truite de ruisseau ; les Alevins ainsi nés se développent en rivièrc et il est certain que si. autour de la frayère, ils trouvent de la nourriturc en abondance, ils y restent assez longtemps, peut être plusieurs années, e1 $\mathrm{y}$ acquièrent la dimension de fertilité de la Truite de ruisseau ; ils se comportent alors généralement comme cette Truite de ruisseau et restent jusqu'au prochain hameçon dans les rivières. Si les Alevins ne trouvent pas ce qu'il leur faut, ou bien en cas de crues, etc..., ils redescendent vite au lac, s'y complaisent dans les eaux superficielles, se nourrissent de plankton et de petites pièces (larves d'insectes), ils s'en gavent littéralement et. toute proportion gardée, se conduisent comme des poulets de Bresse dans leurs mues, ils prospèrent beaucoup et atteignent de belles tailles tout en étant très jeunes relativement. En quelques années, les Truites atteignen! la dimension réglementaire de 30 centimètres, quand elles grossissent encore elles deviennent carnassières, mangeuses de petits poissons ; c'est 
alors que leur fertillité est indiscutable ; il n'est pas possible de dire sûrement si c'est la maturité des éléments sexuels qui motive le changement du régime alimentaire ou le contraire, je croirais assez volontiers à une explication admettant, tout à fait au début, la première raison, puis ensuite la seconde, qui activerait le développement sexuel plus rapide chez les Truites de la catégorie "Truite dé fond " à régime plus nettement carnassier. Il importe donc de voir quelles sont les disponibilités du Lac Léman en matière de frayères, c'est-à-dire en ce qui concerne ses affluents.

Du côté français, sept petites rivières et une grande apportent leurs eaux au lac : la Morge, à Saint-Gingolph - le Torrent, à Lugrin - le Pamphiot; à Anthy - le Redon et le Foron, près de Sciez - le Vion, à Exćenevex, et l'Hermance, audit lieu. La grande rivière est la Dranse. Nombreuses sont les Truites qui, en automne, remontent dans ces rivières pour frayer, les pêcheurs en rivière en prennent tous les ans, mais l'état de ces rivières n'est plus propice à la remontée, il y a des barrages, des radiers, des usines qui interdisent brutalement les remontées, peu de Truites parviennent aux environs des sources. Le Pamphiot, le Redon et le Foron de Sciez, les meilleures de ces rivières, sont de plus très visitées par des amateurs sans scrupules qui capturent aisément les Truites fatiguées par leurs vains efforts pour franchir les barrages.

En ce qui concerne la Dranse, il y a à l'estuaire une entreprise d'extraction de graviers et cáilloux roulés, puis les déversements d'une papeterie et, pour finir, des importants barrages d'usines ; il n'arrive plus un reproducteur dans la Dranse d'Abondance. La Dranse de Bellevaux est barrée par une usine électrique et tout le haut de lā rivière est bel et bien bouché. Des travaux des Services du Reboisement, utiles au point de vue consolidation des rives, réduiront encore plus tard la partie accessible de cette rivière.

Du côté français, la situation serait même absolument désespérée pour les pêcheurs, sans l'activité de la puissante Societé de Peche et de Pisciculture locale qui déverse dans ces cours d'eáu des centaines de milliers d'Alevins chaque année (un tableau succinct figure en note ci-dessous) ( 1 ).

Ceci, avec les reproducteurs emprisonnés dans les hauts bassins, permet de considérer la situation comme passable en ce qui concerne la Truite du lac, car certainement une partie, inappréciable, des Truites produites ou apportées se trouve dérivée vers le lac.

Du côté suisse, nous verrons que la situation n'est pas la même du fait

\begin{tabular}{|c|c|c|}
\hline \multicolumn{3}{|c|}{$\begin{array}{l}\text { (1) Déversements en Truites communes effectués par la Société de Peche } \\
\text { et Pisciculture du Chablais et du Genevois } \\
\text { dans les rivières et affluents directs du lac Léman (Ame et affuents non compris) }\end{array}$} \\
\hline $\begin{array}{l}\text { Ig26: } 62.000 \text { alevins. } \\
\text { 1927: } 10 x .000- \\
\text { rg28:134.000 - }\end{array}$ & $\begin{array}{l}1932: 178.000 \text { alevins } \\
1933: 140.000- \\
1934: 203.000-\end{array}$ & \\
\hline $\begin{array}{l}1929: 194.000- \\
1930: 224.000- \\
1931: 104.000-\end{array}$ & $\begin{array}{l}1935: 209.000- \\
1936: 259.000-\end{array}$ & $\begin{array}{l}\text { quelques sommerlings. } \\
\text { 18.000 sommerlings }\end{array}$ \\
\hline
\end{tabular}


des pêcheries, mais quelques rivières encore bénéficient d'une remonte libre. En gros, elles offrent les mêmes caractéristiques que les rivières savoyardes.

En Suisse, les rivières n'appartiennent pas pour moitié aux propriétaires riverains comme en France, les cours d'eau non navigables, ni flottables ; elles sont propriété des Etats confédérés qui, par conséquent, ont à la fois les droits et les facilités d'y faire ce que leur gouvernement veut.

Le Canton de Vaud, pour ne parler que de celui-là, en a profité pour installer des pêcheries où sont capturées les Truites remontant pour frayer; ces Truites sont pour partie, après fécondation artificielle, remises à l'eau et, pour partie, vendues pour payer les frais ; enfin on profite de la circulation pour débarrasser le lac des Truites portant des caractères de maladies contagieuses. M. Combe a bien voulu me communiquer une sorte d'historique de ces pêcheries, voici ces très intéressants renseignements :

"La première pêcherie installée sur les rives du Léman, fut celle de l'Aubonne à Allaman. Ce n'était pas la première dans le canton ; celle de l'Arnon existait déjà ainsi que d'autres qui ont disparu.

Cétte pêcherie fut installée sur le modèle de celles qui s'exploitaient déjà. On profita du barrage déjà existant au lieudit " la Daille " pour créer la dérivation et construire le bâtiment sur un terrain acheté par l'Etat à cet effet. L'exploitation était confiée à un fermier qui obtenait l'adjudication aux enchères publiques. Il possédait le droit de pêche absolu et réservé dans l'Aubonne depuis le barrage jusqu'au lac.

Ce système de l'affermage fit plàce en 1888 à celui de l'exploitation directe par les agents de l'Etat.

C'est en r 869 que la création de cette pêcherie fut décidée, et l'exécution suivit en 1870 .

La pisciculture et pècherie de la Venoge, à Saint-Sulpice, fut créée pax achat en 1912 et commença à fonctiorner en rgr3.

Les deux installations de l'Aubonne et de la Venoge reposent sur le principe de la trappe à poisson, qui se prend lui-même en franchissant une chambre de capture fermée par une tête de nasse. A Saint-Sulpice, il avait été prévu primitivement, le système de la dérivation avec barrage du lit de la Venoge par des claies mobiles. La capture du poisson n'était plus automatique, mais périodique par la mise à sec du canal de dérivation préalablement grillé vers le bas. Ce système qui fonctionne bien ailleurs, s'adaptait mal à la situation de la pêcherie de Saint-Sulpice et fut abandonné.

Il faut reconnaître que celui de la trappe à poissons ne rend plus comme autrefois, et que les agents affectés à la capture des reproducteurs utilisent de plus en plus la capture directe, à la filoche, au tramail et au lacet (par la queue). C'est ce qui a permis d'utiliser la remontée des Truites au Boiron de Morges et au Boiron de Nyon. Les captures se font, pour cette première rivière, depuis Saint-Sulpice et depuis Promenthoux pour la seconde. C'est ainsi que, depuis l'hiver I932-1933, nous avons pu ajouter Promenthoux au nombre des pêcheries vaudoises du Léman ". 
En tout cas, depuis $1927-\operatorname{rg}^{2} 8$, une moyenne annuelle de 400 Truites environ donnant une moyenne de 64 kilos d'œufs (640.000) a pu être ainsi obtenue. D'une façon générale, l'Etat vaudois cherche à compléter par des achats le total des œufs à i5o kilos environ, soit i .500.000 oufs.

Ces œufs sont ensuite mis comme en pension en montagne, chez des éleveurs à qui les Alevins sont ensuite rachetés par I'Etat, cela encourage l'éleveur à soigner sa production pour obtenir le moindre déchet.

Les Aleving ainsi produits en nombre augmenté de ceux achetés directement, sont déversés dans les rivières. Le bassin du Rhône, qui alimente le Léman, en a reçu :

$$
\begin{aligned}
& 564.000 \text { en } 1933 \\
& 615.000 \text { en } 1934 \\
& 670.000 \text { en } 1935 \\
& 571.000 \text { en } 1936(1) .
\end{aligned}
$$

De plus, certains de ces Alevins, poussés à la dimension de Truitelles, sont déversés, non seulement dans le Léman directement, mais encore une fois dans les eaux libres du bassin du Rhône.

$$
\begin{aligned}
& \text { 10.875 dans le Léman en } 1933 ; 33.115 \text { en eaux libres. } \\
& \begin{array}{lllrl}
\mathbf{5} 5.775 & - & - & 1934 ; 54.105 & - \\
\mathrm{r} 3.625 & - & - & 1935 ; 42.505 & - \\
19.210 & - & - & 1936 ; 40.270 & -
\end{array}
\end{aligned}
$$

En ce qui concerne le canton de Genève, il y a lieu de noter que le service forestier génevois commence à imiter le canton de Vaud et a pu produire, durant l'hiver 1936-1937: 45.000 oufs de Truite de lac.

Les mises à l'eau sont les suivantes :

$$
\begin{array}{r}
\text { Alevins } \text { Truites. } \\
\text { I932 }^{3} \mathbf{3 2 5 . 0 0 0} \\
\text { 1933: } 222.500 \\
\text { 1934: } 470.000 \\
\text { 1935:510.000 } \\
\text { I936 : } 251.000
\end{array}
$$

tant dans le Lac et la Versoix ( 35 \% environ), que dans le bassin du Rhône en aval de Genève.

$$
\begin{aligned}
& \text { Sommerlings Truites. } \\
& \text { r932 : } 19.500 \\
& 1933: 18.900 \\
& 1934: 25.900 \\
& \text { I } 935: 29.300 \\
& 1936: 25.100
\end{aligned}
$$

Mais la disposition des vannes du Rhône à Genève laissant la possibilité de remonte et le Rhône se trouvant en fait coupé à l'aval de Bellegarde,

(r) Chiffres extraits des comptes rendus du Conseil d'Ftat Vaudois, communiqués par M. l'Inspecteur cantonal Grivsz et ses services. 
l'apport fait dans les rivières en aval de Genève n'est pas sans intérêt pour le lac.

Les forestiers du canton du Valais déversent naturellement toute leur production dans les rivières qui apportent leurs eaux au Haut-Rhône, il est certain de plus qu'une bonne partie des immersions se fait en Truite arc-en-ciel.

Voici en tous cas les chiffres :

Production en oufs de Truites.

$$
\begin{aligned}
& \text { 193 г : } 300.000 \\
& 1932: 445.000 \\
& \text { 1933 : } 335.000 \\
& \text { rg34 : } 440.000 \\
& \text { } 935: 380.000 \\
& \text { I936 : } 500.000
\end{aligned}
$$

$$
\begin{aligned}
& \text { 1931 : 320.850 alevins. }-8.300 \text { truitelles } \\
& 1932: 666.000 \quad-\quad-22.800 \quad- \\
& 1933: 314.000-2.300- \\
& \text { 1934:362.000 - }-28.200- \\
& 1935: 414.500-8.100- \\
& \text { 1936:413.500 - }-7.200-
\end{aligned}
$$

Le canton du Valais achète donc des œufs pour augmenter ses déversements.

Il y a lieu de souligner ces efforts véritablement intéressants ; si, d'après ces données, il est impossible de dire que le chiffre annoncé plus haut de 200.00o Alevins est effectivement mis chaque année au lac, il est manifeste que, grâce aux Services forestiers suisses, en particulier le Service Vaudois, la chute qui risquerait d'être rapide se trouve véritablement freinée, mais la baisse de production n'en est pas moins montrée par les chiffres des statistiques.

Et cela fait que le Léman est encore un lac relativement riche en Truites, il suffit pour s'en apercevoir de voir ce qui se passe dans quelques autres lacs.

D'abord, dans le Lac de Constance qui, par sa superficie, approche du Léman ; je n'ai malheureusement pas des statistiques complètes, mais les renseignements suisses sont assez éloquents :

En 1930, sur 175.300 kilos de poissons pêchés (chiffres arrondis).

$$
\begin{aligned}
& \text { I } 93 \text { I, }-122.400 \\
& \text { I } 932,-110.700 \\
& \text { I } 933,-214.500 \\
& 1934,-255.000 \\
& 1935,-155.300 \text { - }
\end{aligned}
$$

la Truite représente : 


$$
\begin{array}{rrrr}
\text { En } 1930, & 2.087 & \text { kilos, soit } 1,2 & 1 \\
1931, & 1.505 & - & 1,2 \% \\
1932, & 2.144 & - & 1,9 \% \\
1933, & 2.568 & - & 1,2 \% \\
1934, & 2.033 & - & 0,7 \% \\
1935, & 2.033 & - & 1,0 \%
\end{array}
$$

I I.990 kilos, soit en moyenne I \%.

Il est vrai que dans ce lac ce ne sont pas les espèces communes qui ont la majorité, en effet :

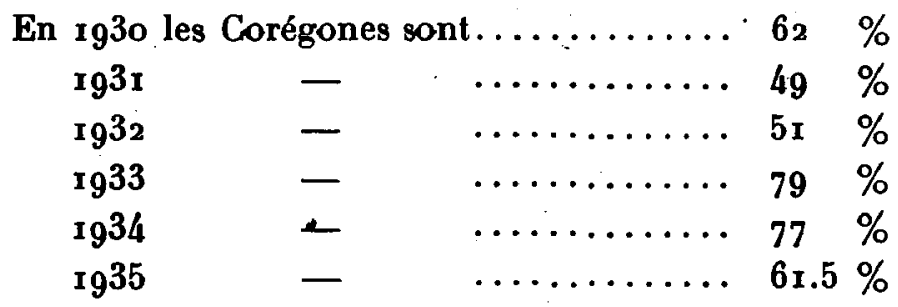

du total, soit en moyenne : $63 \%$.

Tandis que Perches, Lottes et Sandres ne représentent en moyenne que 26.000 killos (16.000-32.000) soit : $15 \%$.

Le lac de Neuchatel donne, pour les trois dernières années, dont je possède les statistiques :

et en moyenne : $2,4 \%$.

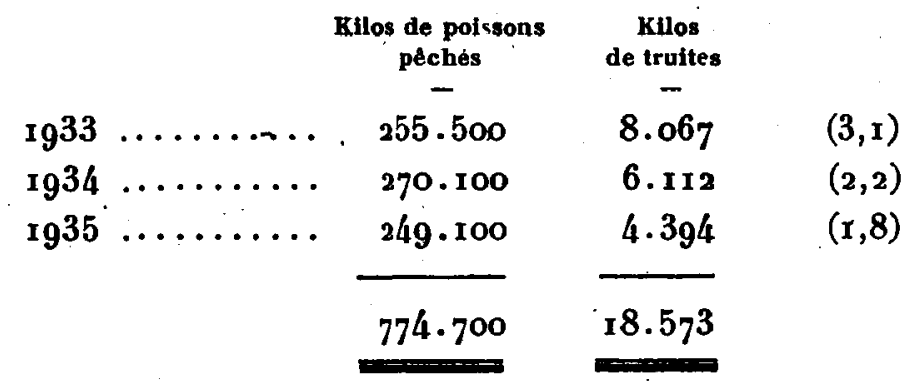

Les Corégones étant :

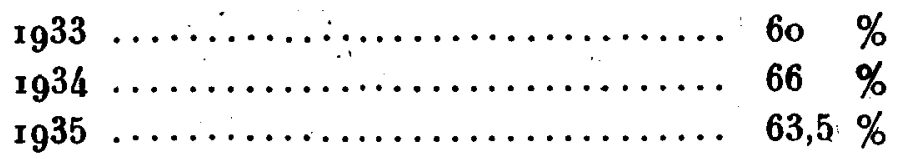

soit en moyenne également $63 \%$, et les Perches et Lottes en moyenne : $17 \%$.

Et le lac des Quatre Cantons, pauvre en produits globaux et qui, par certains côtés, ressemble beaucoup au Léman : 


\begin{tabular}{|c|c|c|c|c|}
\hline & & $\begin{array}{c}\text { Kilos de poissons } \\
\text { péches } \\
\text { chiffres arrondis } \\
-\end{array}$ & $\begin{array}{c}\text { Kilos } \\
\text { de } \\
\text { truites } \\
-\end{array}$ & \\
\hline $\mathrm{Ig}_{2} / 33$ & $\ldots \ldots$ & $\overline{5}_{7} \cdot 100$ & I. 539 & $(2,6)$ \\
\hline I $933 / 34$ & $\ldots \ldots \ldots$ & $47 \cdot 700$ & I. 467 & $(3,0)$ \\
\hline I $934 / 35$ & $\ldots \ldots \ldots$ & 36.000 & 2.045 & $(5,6)$ \\
\hline $\lg 35 / 36$ & $\ldots \ldots$ & 40.600 & I . 682 & $(4, I)$ \\
\hline & & $181 \cdot 400$ & 6.733 & \\
\hline
\end{tabular}

et en moyenne : $3.7 \%$.

Les Corégones étant :

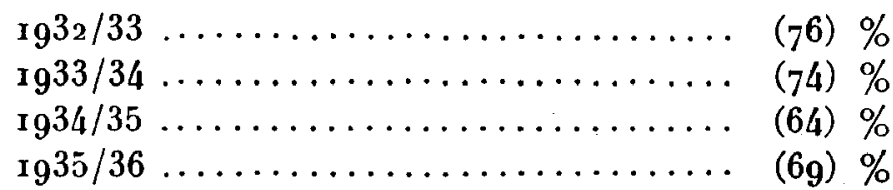

soit en moyenne 7r \% et les Perches et Lottes en moyenne : $6 \%$ seulement.

L'emploi de mesures conservatoires nouvelles semble tout de même s imposer dans le Léman. Avant toute chose, il s'agit de savoir si, oui ou non, la presque totalité des Truites vivant dans le lac est de la catégorie "Truite argentée " vraiment stérile et si, par conséquent, les pêches de lève sont des pêches sans répercussion fâcheuse pour le dit peuplement, ou bien si les "Truites argentées " ne sont, pas plus que les autres, démunies de facultés de reproduction et sont à protéger efficacement.

Mettant à profit la documentation pratique traitant de ce sujet, je dois, avant d'entrer dans le problème de la stérilité de la "Truite argentée ", liquider une petite question qui, au moins épisodiquement n'est pas sans intérêt, c'est celle de savoir si la "Truite de lac " va obligatoirement en rivière pour frayer. Je dois, à la vérité, dire que Neresheimer affirme que, dans certaisn lacs d'Autriche, petits lacs d'ailleurs comme l'Ammersee, il est prouvé que la Truite de lac fraye, non dans les ruisseaux arrivant à ce lac, mais dans le fond même à l'emplacement de sources sous lacustres ; mais il est certain que cette adaptation est assez spéciale et que, dans les grands lacs, tout en ne niant pas la possibilité de la chose, le fait ne doit être qu'extrêmement isolé et sans importance pour le repeuplement.

La grande question est de savoir si oui ou non la "Truite argentée " est destinée à rester toujours stérile. La question doit se développer en deux temps :

D'abord - y a-t-il dans les lacs des cas de stérilité permanente certaine sur des Truites? J'estime que c'est, non seulement possible, mais pro-

(1) Documentalion extraile de Ja revue: Schweizerisde Fischerci Zeitung, Berne ; du Bulletin suisse de péche et de pisciculture, Neuchàtel, et du journal Le Pêcheur Suisse, Lausanne. 
bable. Pour diverses raisons : en particulier impossibilité matérielle de remonter, des Truites femelles peuvent très bien, au lieu d'abandonner leur ponte, conserver jusqu'à résorption dans le corps les œufs produits, l'organisme en profitant d'ailleurs ; la Truite garde son aspect de plénitude somatique, c'est-à-dire l'aspect de "Truite argentée ", les organes sexuels anormalement maintenus dans des conditions de distension et gêenés pour une ovulation ultérieure, peuvent très bien alors dégénérer au point de donner des inféconds ultérieurs. Il s'agit là, naturellement, d'individualités et c'est pour cela qu'il n'y a pas lieu de mettre en doute les résultats de certaines autopsies faites sur de gros sujets vraiment stériles; mais il doit s'agir, dans ce cas, de gros sujets, et si les pêcheurs de lève voulaient arguer de cette stérilité pour continuer leur pêche, ils devraient être logiques avec eux-mêmes et adopter, pour ne pêcher que ces Truites, des filets à très grosses mailles $: 50 \mathrm{~m} / \mathrm{m}$ au moins. Ce n'est pas le ćas.

La plupart du temps, - au contraire, il paraît à peu près certain que la stérilitẻ n'est qu'une forme temporaire ou bien qu'elle n'est que transitoire. - Temporaire $P$ dans le cas d'individus jeunes quoique gros. - Transiloire ? dans ce cas il s'agit de Truites qui ayant frayé l'année précédente sont comme en repos général pendạnt un ou deux hivers avant de recommencer à donner des produits sexuels. Je dois faire connattre que les résultats de quelques autopsies ou constatations faites à Thonon, me permettent de dire que, sur des Truites d'entre 400 et 760 grammes, j'ai trouvé des ovaires existants mais comparables en importance à ceux de Sömmerlings ou Truiteliles de deux étés -; en un mot des organes existants, paraissant capables de fonctionner plus tard, mais n'ayant certainement pas encore produit.

Le poids critique des Truites n'est pas fixe et mes constatations ne permettent que de donner une moyenne : - de poids : 750 grammes ; d'épaisseur dè corps : 8 centimètres - et d'âge : 5 ans. Des expériences ont êté faites à plusieurs reprises déjà, mais mériteraient d'être continuées. - Hofen a trouvé que des Truites de lac, suivies dans un bassin, perdaient leur aspect argenté, mais restaient stériles, peut-être a-t-il là trop grande modification d'habitat ou bien expérience interrompue trop tôt ; cliamétralement opposés sont les résultats de HaAxh qui a examiné près de zoo Truites de lac argentées, et a conclu que le pourcentage de fertilité était de 0 . \% pour les sujets de 3 à 4 ans, 5,7 \% pour ceux de 5 ans, $22 \%$ pour ceux de 6 ans, too\% pour ceux de 7 ans et à nouveau o \% pour les plus vieux. Je sais bien qu'on reproche à cette expérience d'avoir été faite sur trop peu de sujets âgés, mais je suis heureux de constater que les conclusions sont à peu près les mêmes que celles qu'il m'a été donné de faire, sans avoir eu connaissance alors des résultats de HaAKB.

J'ai aussi à citer une expérience personnelle, qui a eu pour sujets des Truites en stabulation à l'Etablissement de Thonon, à vrai dire l'expérience n'a pas porté sur des Truites de lac trop délicates à maintenir long- 
temps en captivité, mais sur des Truites arc-en-ciel ; il n'est donc pas possible de parler d'un changement brusque du mode de vie.

L'abondance et la régularité de l'alimentation avaient amené la création, parmi des "Arc-en-ciel " de deux étés, d'une véritable variété de Truites arc-en-ciel argentées à livrée brillante et métallique. Ces Poissons étaient tous de plus forte taille que leurs contemporains à livrée ordinaire. Ces Truites ont été suivies, elles se sont révélées en bloc stériles à trois ans, (hiver I932-1933) Jes normales étant déjà fertiles. A quatre ans (hiver r933-r934) un certain nombre de ces Truites avait déjà beaucoup perdu de son éclat et quelques-unes se sont montrées fertiles (à vrai dire peu fertiles) mais surtout des mâles avaient déjà nettement de la laitance. Durant l'hiver 1934-1935, à 5 ans, les livrées de toutes ces Truites étaient devenues à peu de chose près normales et la stérilité avait pratiquement totalement disparu, tant chez les femelles que chez les mâles.

Pour ces Truites, dans une situation physique très voisine de celle des Truites argentées, il s'est donc vérifié que la stérilité n'était que temporaire et qu'elle disparaissait avec l'affaiblissement, d'ailleurs progressif, du caractère brillant.

Je crois l'expérience assez concluante, elle peut être refaite facilement même sur des Truites en liberté dans un lac pas trop grand, à l'aide d'individus marqués dont on pourrait suivre l'évolution.

La question de la stérilité des Truites argentées est donc, à mon avis, à résoudre par la négative ; il n'y a, sauf quelques cas particuliers, qu'une adolescence très prolongée et plantureuse et une maturité sexuelle retardée.

Je n'ai pas de constatations personnelless concernant les Truites stériles transitoires, c'est-à-dire en période de repos après première fraye.

Pour conserver en quantité suffisante le peuplement en Truites du lac Léman et assurer, à la fois des pêches rémunératrices d'une espèce de qualité, il y a du côté français certaines mesures à appliquer :

Il est d'abord impérieusement indiqué de supprimer la pêche d'hiver qui détruit bon nombre de poissons sans leur avoir laissé le temps de frayer, d'ailleurs chose à signaler, la Truite à ce moment est nettement dépréciée, sur le marché à Genève la Truite qui valait d'Avril à Septembre i 936 en francs suisses non dévalués : 8 fr. 85 le kilog. n'était plus coté en Janvier que $8 \mathrm{fr}$. suisses dévalués.

Mais la pêche d'hiver ou de lève a, encore, parmi les pêcheurs, quelques adeptes acharnés, la masse des pêcheurs est indifférente ou hostile, et il est malaisé de persuader les dits adeptes du rôle néfaste pour le lac qu'ils persistent à vouloir continuer à jouer, ils argutient d'ailleurs en indiquant la nécessité et l'intérêt pour eux de pouvoir mettre en vente des Truites pour les fêtes de Noël et du Nouvel An. On peut leur répondre que la consommation est assez faible pourtant et que cela justifierait, tout au plus, deux, pêches, une avant chaque fête, et non pas des pêches continuelles ; ils invo- 
quent l'obligation pour eux de gagner leur vie à la fin d'une longue période dé pêches réduites et restreintes.

Je ne prétends pas qu'ils aient tout à fait tort, mais on peut, tout de même, leur faire remarquer qu'avec un grain d'idée de prévoyance et d'économie, alors que la pêche rend bien et que le poisson se vend avantageusement c'est-à-dire en été, ils pourraient mettre de côté des sommes qui pourraient être au moins égales à ce que la pêche de lève peut rapporter, avec ses indiscutables aléas : tempêtes, pertes de filets, coups nuls et gêne des nuits avec lune. Quelques années de fréquentation des pêcheurs ne me permettent pas d'espérer les voir venir à cette pratique, il conviendrait donc d'échafauder une réglementation donnant satisfaction aux pêcheurs, mais ne causant pas des dégâts importants, l'augmentation de la dimension des mailles des filets ne serait pas facilement acceptée par les pêcheurs qui prétendent que cela obligerait à engager beaucoup de matériel pour des prises somme toute minimes. Cette augmentation mettrait pourtant à l'abri les Truites qui sont à la période de passage, mais il serait assez logique de n'autoriser qu'une pêche par semaine durant la période 21 Décembre3r Janvier; à défaut de l'interdiction complète de la lève je crois qu'il faudra bien en venir là comme demi-mesure, à défaut de mesure complète.

Il faudrait aussi contribuer, comme les Suisses, au réempoissonnement en Truites du Lac. G'est chose qui pourra devenir facile puisqu'il y a tout lieu d'espérer que les établissements de pisciculture en construction un peu partout en Savoie peuvent laisser entrevoir qu'un jour l'Etablissement de Thonon n'aura plus comme but la fourniture d'alevins aux Sociétés de pècheurs en rivières de la Savoie et de la Haute-Savoie, mais pourra se consacrer beaucoup plus activement; à la pisciculture du lac. L'acquisition de quelques centaines de milliers d'oufs de Truite commune compensera l'impossibilité d'établir' en France des pécheries sur les rivières et les alevins seraient poussés jusqu'au stade de truitelles dans les bassins qui pourront être dégarnis de beaucoup de reproducteurs conservés. Je vois là, en ce qui concerne l'avenir de la Truite dans le Léman, un facteur d'espérance et parfaitement réalisable, vu l'essor imprimé par le Sous-Secrétaire d'Etat à l'Agriculture à la création d'Etablissements de pisciculture en Savoie.

Enfin il $\dot{y}$ aura lieu, et cela non seulement en faveur des Truites, mais aussi des Corégones et de l'Omble-Chevalier, de limiter l'extension de la Perche et de la Lotte, extension qui prend le caractère d'une 'occupation de plus en plus totale : $49 \%$ des captures de $r 936$.

Contre la Lotte les mesures à prendre sont difficiles à envisager, sa pêche est, en effet, déjà permise en toutes saisons et la mesure réglementaire est déjà si faible qu'il n'est vraiment pas possible d'en envisager une diminution. Peut-être arriverait-on à quelque chose en délivrant gratuitement des licences aux pêcheurs, à charge de se consacrêr uniquement à cette pêche sans limitation du nombre des engins, et comme la Lotte n'est pas un poissón de valeur alimentaire négligeable, peut-être serait-il indiqué pour 
l'Etat, de subventionner une petite usine saisonnière, où pourrait se faire la mise en conserves de ce poisson.

Contre Ia Perche, la solution apparaît plus aisée (sans la moindre intervention il y a d'ailleurs quelquefois des maladies spéciales à cette espèce qui portent un sérieux coup à cette population). Parmi les mesures artificielles j'estime que :

$I^{\circ}$ la pêche de la grosse Perche, j'appelle ainsi celle qui dépasse $20 \mathrm{~cm}$., pourrait être autorisée en toutes saisons même en mai, avec des engins à mailles de $26 \mathrm{~m} / \mathrm{m}$, cela éliminerait déjà bon nombre de reproducteurs ayant des qualités marchandes indéniables ;

$2^{\circ}$ ensuite les onze autres mois de l'année pourrait être autorisé l'emploi de nasses à mailles de $22 \mathrm{~m} / \mathrm{m}$ seulement, beaucoup de Perches de la dimension légale $15 \mathrm{~cm}$, qui échappent aux nasses à mailles de $25 \mathrm{~m} / \mathrm{m}$ seraient ainsi capturées. Il ne serait d'ailleurs pas question de réduire la taille réglementaire de $15 \mathrm{~cm}$. La reproduction de cette espèce serait ainsi petit à petit réduite mais non supprimée, il n'est pas question d'obtenir la disparition d'une espèce qui au point de vue alimentaire a ses qualités intrinsèques, mais qui est vraiment à l'heure actuelle trop encombrante dans. le lac. L'emploi de montes à mailles plus restreintes que celles actuellement permises est aussi à prohiber ; car le filet endommage le poisson qui souffre ainsi, même si de trop petite taille il est en définitive rejeté au lac, tandis que la nasse ne meurtrit guère ses captures et ne retient pas les animaux nettement trop petits.

Ma conclusion, je puis la formuler en quelques lignes :

La Truite de lac dans le Léman existe encore en quantité suffisante pour rétablir en sa faveur une situation actuellement menacée ; elle ne pourra ètre efficacement protégée que par une suppression ou tout au moins une importante réduction de la pêche d'hiver dite de lève, par une augmentation des déversements de Truites du fait de l'entrée en jeu de déversements français et enfin par une lutte implacable contre la lotte et les mesures sérieuses contre le développement abusif de la Perche. 